\title{
Structure and Electronic Properties of a-Si:H Investigated with Quantum Simulation
}

\author{
Haili Li ${ }^{1}$, Mitsuhiro Matsumoto ${ }^{1}$ \\ ${ }^{1}$ Graduate School of Engineering, Kyoto University \\ Kyoto-daigaku-Katsura, Kyoto 615-8540, Japan \\ li.haili.75x@st.kyoto-u.ac.jp; matsumoto@kues.kyoto-u.ac.jp
}

\begin{abstract}
In order to investigate various properties of hydrogenated amorphous silicon (a-Si:H) for improvement of low conversion efficiency of solar cells, a series of quantum simulations based on the density functional theory combined with the tight binding model were performed for a-Si:H with various hydrogen concentrations and fabrication methods. The radial distribution function (RDF) for SiSi pairs indicates that samples with higher $\mathrm{H}$ concentration (20\% and $25 \%$ ) give a better structure, but the RDF of Si-H pairs suggests that samples with lower $\mathrm{H}$ concentration (14\% and 20\%) may give better properties. The coordination number analysis indicates that more defects (dangling bonds and floating bonds) exist in $25 \%$ and $20 \% \mathrm{H}$ concentration samples. Overall, a-Si:H with $14 \% \mathrm{H}$ concentration gives most preferable structure. Samples with slower cooling rate show slightly better structure.
\end{abstract}

Keywords: hydrogenated amorphous silicon, structural analysis, electronic property, DOS analysis, solar cell, quantum simulation

\section{Introduction}

Hydrogenated amorphous silicon (a-Si:H) is a low-cost and excellent light-absorbing material and has attracted much attention in the field of thin-film solar cells. However, it still has shortcomings such as low conversion efficiency and lightinduced degradation (Staebler-Wronski effect) [1]. In order to improve its total performance, further research is still needed on microscopic structure and basic performance. The disorder in the microstructure of hydrogenated amorphous silicon has led to the complexity of its structure. In the past 40 years, a lot of theoretical investigation has been done, from simple classical models to various types of quantum simulations.

For example, Luo et al. adopted molecular dynamics methods to simulate the influence of different substrate temperatures on the structure of a-Si:H [2], showing that the surface roughness of a-Si:H films decreases as the substrate temperature increases. Czaja et al. reported the structure, electronic and optical properties of different sizes of a-Si:H based on a first-principle density functional theory (DFT) [3]. Zhang et al. also executed first-principle DFT calculations to study a network growth in films [4], showing a continuous disorder-order phase transition.

Among various factors for the performance of a-Si:H, hydrogen concentration is one of the most relevant ones. Legesse et al. investigated the optical gap and the electron mobility with VASP [5]. They proposed three regimes of the hydrogen concentration; in the unsaturated regime, both the optical gap and the mobility increase with the increase of hydrogen concentration until they converge to some constant values in the saturated regime. Ukpong et al. also found with tight-binding (TB) quantum simulations [6] that the local order and the intrinsic stress are much affected by the hydrogen concentration, suggesting the existence of optimal concentration.

To look into the structure on atomic scales and to further improve the photoelectric conversion efficiency, we have been developing an atomic model. In this paper, we report the structural and electronic properties investigated with quantum simulations.

\section{Methods}

We have adopted a molecular dynamics (MD) simulation with a density functional tight binding (DFTB) model, utilizing the DFTB+ package [7] with the matsci-0-3 parameter set [8].

To generate the atomic configuration of a-Si:H at a given hydrogen concentration, we employed the heat and quench method similar to Ref. [9]. First, a cubic super-cell of Si crystal with 64 atoms in $10.862 \AA \times 10.862 \AA \times 10.862 \AA$ is created 
a given number of randomly selected $\mathrm{Si}$ atoms are replaced by hydrogen atoms. The system is heated well above the melting temperature $(2370 \mathrm{~K})$ with a temperature controlled molecular dynamics (MD) simulation. After the high temperature being kept for 3ps, the melt system is cooled down to $300 \mathrm{~K}$. We investigated the cases with six different $\mathrm{H}$ concentrations and two cooling rates as follows. (i) H concentration: 8\% (5 atoms), $11 \%$ (7), 14\% (9), 17\% (11), 20\% (13), and $25 \%$ (16). (ii) Cooling rate: fast cooling with $0.414 \mathrm{~K} / \mathrm{fs}$ and slow with $0.138 \mathrm{~K} / \mathrm{fs}$.

The periodic boundary conditions are assumed for all directions. For the electronic energy evaluation, we use a $2 \times 2 \times 2$ Monkhorst-Pack mesh for the Brillouin zone sampling. The time step the MD simulation is $1.0 \mathrm{fs}$

\section{Results and Discussion}

\subsection{Influence of Hydrogen Concentration}

To investigate the sample structure, the radial distribution function (RDF) for Si-Si pairs is evaluated, as shown in Fig. 1 (a). It generally shows an amorphous character. As the $\mathrm{H}$ concentration increases, the local order indicated by the first peak becomes more apparent. However, the second and the third peaks gradually decrease, suggesting that the midrange order is weakened by the hydrogen increase. The dependence is not apparent in Si-H RDF shown in Fig. 1 (b).
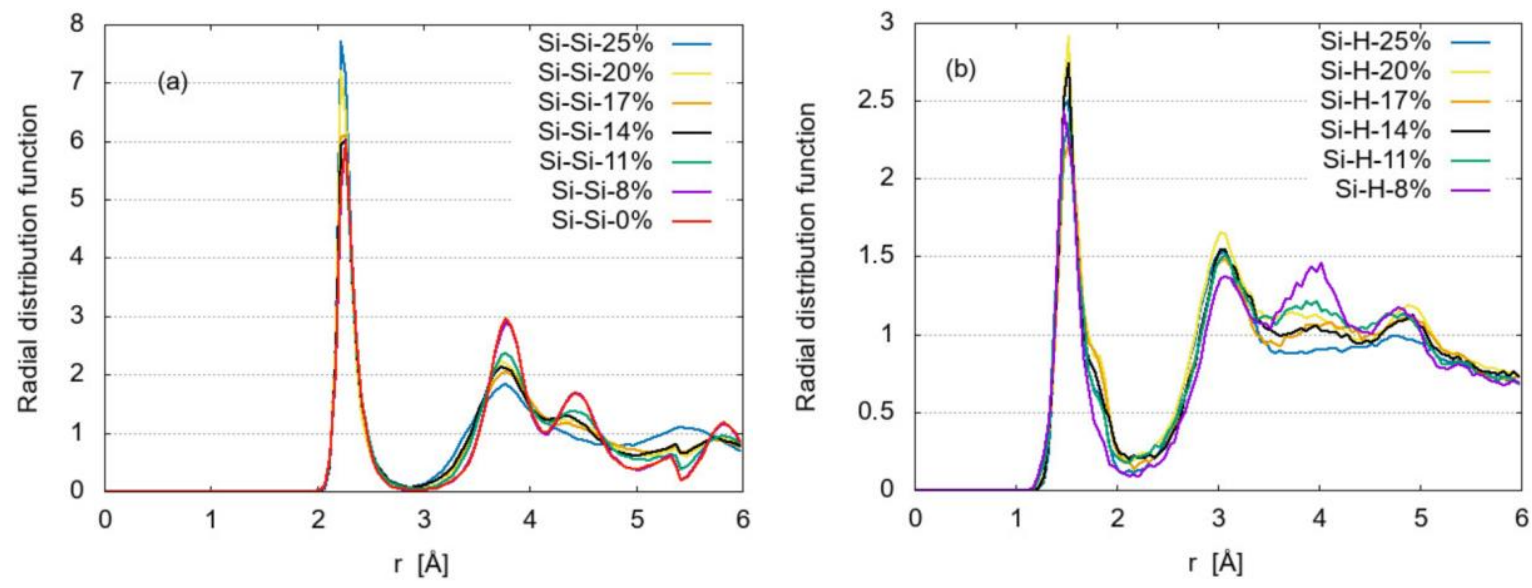

Fig. 1: Radial distribution function for fast cooling samples: (a) $\mathrm{Si}-\mathrm{Si}$, (b) $\mathrm{Si}-\mathrm{H}$.

The mean distance between the nearest Si-Si pairs is $2.2 \AA \sim 2.3 \AA$, while that for the second neighbour is $3.7 \AA 23.8$ $\AA$. This is in good agreement with previous results [4]. These distances are little affected by the hydrogen concentration, indicating that the basic Si network remains intact. 
Table 1: Atomic coordination of a-Si:H; fast cooling sample.

\begin{tabular}{c|rrrrrrr|c}
\hline $\mathrm{H}$ concentration & \multicolumn{1}{|c}{$0 \%$} & \multicolumn{1}{c}{$8 \%$} & \multicolumn{1}{c}{$11 \%$} & \multicolumn{1}{c}{$14 \%$} & \multicolumn{1}{c}{$17 \%$} & \multicolumn{1}{c}{$20 \%$} & \multicolumn{1}{c}{$25 \%$} & Experiment [10] \\
\hline $\mathrm{Si}_{5}$ (floating) & $0.4 \%$ & $0.3 \%$ & $0.5 \%$ & $2.6 \%$ & $2.2 \%$ & $0.4 \%$ & $1.8 \%$ & \\
$\mathrm{Si}_{4}$ & $78.6 \%$ & $77.6 \%$ & $70.5 \%$ & $64.8 \%$ & $58.8 \%$ & $45.4 \%$ & $45.9 \%$ & \\
$\mathrm{Si}_{3}$ (dangling) & $20.9 \%$ & $13 \%$ & $14.1 \%$ & $12.5 \%$ & $14.9 \%$ & $24.2 \%$ & $21.7 \%$ & \\
$\mathrm{Si}_{2}$ (dangling) & $0.1 \%$ & $0.1 \%$ & $1.0 \%$ & $1.0 \%$ & $1.2 \%$ & $0.5 \%$ & $0.6 \%$ & \\
$\mathrm{Si}_{4} \mathrm{H}$ & & $0.1 \%$ & $0.3 \%$ & $0.9 \%$ & $0.4 \%$ & $1.0 \%$ & $1.0 \%$ & \\
$\mathrm{Si}_{3} \mathrm{H}$ & & $8.8 \%$ & $12.9 \%$ & $16.9 \%$ & $19.2 \%$ & $25.1 \%$ & $26.7 \%$ & \\
$\mathrm{Si}_{2} \mathrm{H}$ & & $0.1 \%$ & $0.4 \%$ & $1.2 \%$ & $2.5 \%$ & $2.6 \%$ & $1.6 \%$ & \\
$\mathrm{Si}_{x} \mathrm{H}_{2}$ & & 0 & $0.1 \%$ & $0.1 \%$ & $0.6 \%$ & $0.8 \%$ & $0.6 \%$ & \\
\hline$N_{c}$ & 3.793 & 3.871 & 3.834 & 3.877 & 3.814 & 3.728 & 3.773 & 3.88 \\
\hline
\end{tabular}

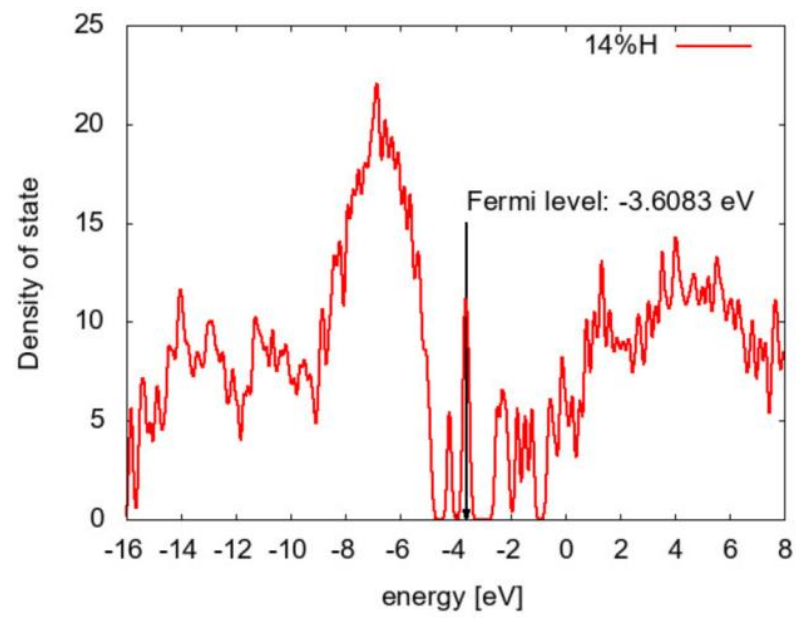

Fig. 2: DOS for the fast-cooling sample with $14 \% \mathrm{H}$ concentration.

The atomic coordination number, i.e., the number of nearest neighbours around a $\mathrm{Si}$ atom, is evaluated. As shown in in Table 1, the perfect four coordination $\left(\mathrm{Si}_{4}\right)$ dominates of course, followed by and $\mathrm{Si}_{2}$. The average coordination number $N_{c}$ fluctuates around $\sim 3.8$, and the concentration dependence is not apparent. The largest $N_{c}$ is obtained for the $14 \%$ concentration sample, which is close to an experimental value 3.88 [7]. One possible reason for the large $N_{c}$ is the existence of "floating bonds" indicated by $\mathrm{Si}_{5}$.

The electronic state analysis was done to obtain the density of states (DOS). An example is shown in Fig. 2 for the sample with $14 \%$ hydrogen concentration. There exist two apparent groups of defect states in the band gap $(-4.6 \mathrm{eV}<E<$ $-2.8 \mathrm{eV})$. Although strict assignment is not possible yet, these should correspond to the dangling bond states and the floating bond states.

\subsection{Influence of Cooling Rate}

The RDFs are compared in Fig. 3 between the fast cooling $(0.414 \mathrm{~K} / \mathrm{fs})$ and the slow cooling $(0.138 \mathrm{~K} / \mathrm{fs})$ samples with the same $\mathrm{H}$ concentration (14\%). Although the apparent structure is almost the same, the height of peaks indicate that the ordering is more developed for the slow cooling sample. 


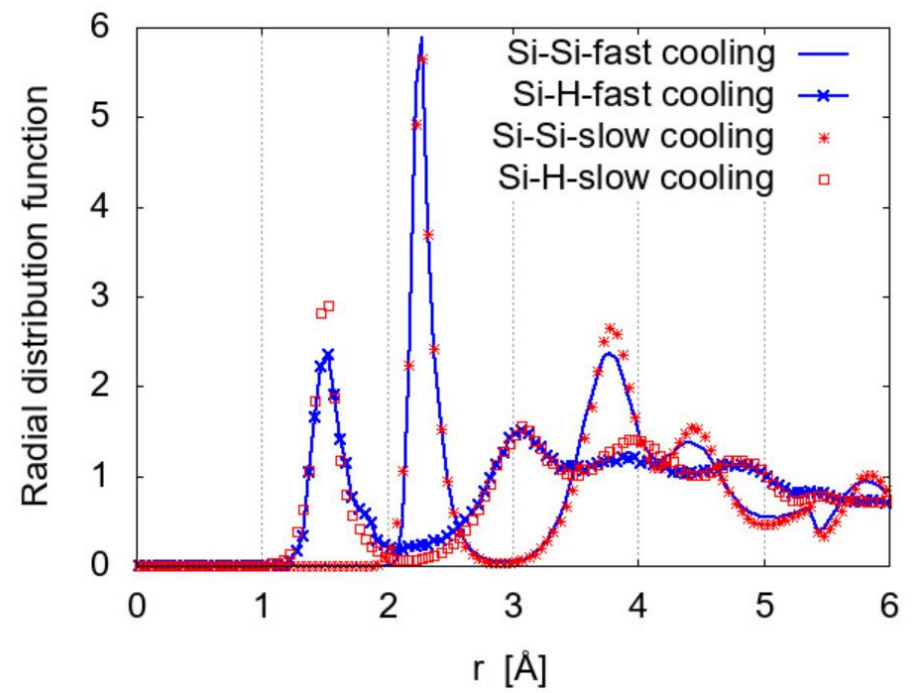

Fig. 3: RDF of $14 \% \mathrm{H}$ concentration samples with different cooling rates.

\section{Conclusion}

We investigated the structure and the electronic properties of hydrogenated amorphous silicon with a quantum simulation, focusing on the effects of the hydrogen concentration and the cooling rate. The radial distribution function the coordination number analysis indicate that the hydrogen concentration strongly affects the structure. The sample $\% \mathrm{H}$ concentration has a largest coordination number, probably caused by the balance between the numbers of the bonds and the floating bonds. The cooling rate has little effect on the structure, but the sample with slower cooling rate slightly more structured.

\section{References}

[1] R. A. Street, Hydrogenated amorphous silicon, Cambridge Univ. Press. 1991.

[2] Y. Luo, H. Gong, N. Zhou, H. Huang and L. Zhou, "Molecular dynamics study about the effect of substrate temperature on a-Si:H structure," Appl. Phys. A, 124, 18, 1-9, 2018.

[3] P. Czaja, M. Celino, S. Giusepponi, M. Gusso, and U. Aeberhard, "Ab initio study on localization and finite size effects in the structural, electronic, and optical properties of hydrogenated amorphous silicon," Comput. Mater. Sci. 155, 159168,2018

[4] Y. Zhang, H. Wang, Y. Gou, and S. Jiang, "Evolution of medium-range order and surface compositions by mechanism driven model with realistic network," Appl. Surf. Sci., 464, 321-327, 2019.

[5] M. Legesse, M. Nolan and G. Fagas, "Revisiting the dependence of the optical and mobility gaps of hydrogenated Amorphous Silicon on Hydrogen Concentration," J. Phys. Chem. C, 117, 23956-23963, 2013.

[6] A. M. Ukpong, M. Harting and D. T. Britton, "Theoretical study of strain fields and local order in hydrogenated amorphous silicon," Phil. Mag. Lett., 88, 4, 293-302, 2008.

[7] https://www.dftbplus.org/

[8] https://www.dftb.org/parameters/download/matsci/matsci-0-3-cc/

[9] N. C. Cooper, C. M. Goringe, and D. R. McKenzie, "Density functional theory modelling of amorphous silicon," Comput. Mater. Sci. 17, 1-6, 2000.

[10] K. Laaziri, S. Kycia, S. Roorda, M. Chicoine, J. L. Robertson, J. Wang, and S. C. Moss, "High energy X-ray diffraction study of pure amorphous silicon," Phys. Rev. B, 60, 13520-13533, 1999. 